\title{
Epigenetics of the immune system and alterations in inflammation and autoimmunity
}

\section{"Epigenetics plays a key role in the etiology of many other complex diseases including autoimmune and inflammatory diseases."}

First draft submitted: 20 February 2017; Accepted for publication: 20 February 2017; Published online: 3 April 2017

As guest editors, we are delighted to present you the second special issue of Epigenomics focusing on the epigenetic regulation of the immune system and stromal cell compartments and their alterations in autoimmune, allergic and other inflammatory diseases.

While initial efforts have concentrated on the frequent and large-scale changes of the epigenome in cancer in particular DNA methylation, research in the last decade indicates that epigenetics plays a key role in the etiology of many other complex diseases including neurodegenerative, psychiatric and metabolic disorders, as well as autoimmune and inflammatory diseases. The immune system with its high demands on plasticity and reactivity toward internal and external cues is a paradigm for epigenetic regulation. Furthermore, environmental factors that are known to exert disease promoting or protecting effects mediate their effects through changes in the epigenome in various inflammatory diseases.

Several large-scale consortia have been initiated which create publicly available highresolution maps of the different levels of the epigenome for immune disease-relevant cells including the USA-lead ROADMAP project and the EU-funded BLUEPRINT project in the framework of the International Human Epigenome Consortium. BLUEPRINT has recently published a number of high-profile studies, some of which investigated the natural variability of the epigenome of immune cells as well as changes during the differ- entiation and maturation of different cell lineages and their alterations in hematopoietic malignancies, as summarized in a commentary by Beck and Ecker in this issue of Epigenomics [1].

Firestein and colleagues review in this special issue the changes to the different levels of the epigenome in fibroblast-like synoviocytes in rheumatoid arthritis and illustrate the insights gained by integrating epigenome analyses [2]. The epigenome can provide important clues on how genetic variants influence the susceptibility and course of the disease. Gay and colleagues review in this issue the current knowledge on the complex interplay between genetic and epigenetic variation in rheumatoid arthritis. Gay and colleagues underline the importance of considering multiple levels of epigenetic modifications together with the three-dimensional architecture of the genome in disease-relevant tissues or cell types to identify novel disease pathways and therapeutic targets [3]. DNA methylation signatures can potentially be used to predict the response to disease modifying antirheumatic drugs as exemplified by the epigenome-wide association study (EWAS) by Fryer $e t$ al., in which the authors identified a $2 \mathrm{CpG}$ signature that predicted treatment response in early rheumatoid arthritis [4].

While most of the research has focused on the analysis of DNA methylation changes in immune-related diseases, prominent changes also occur at the level of histone modifica-
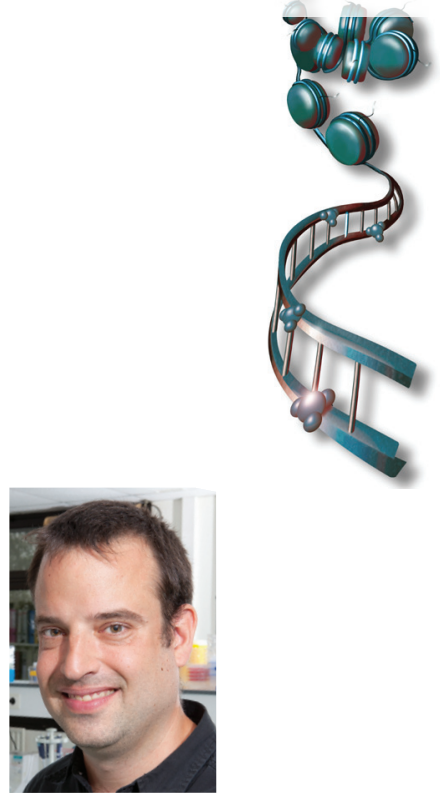

Jorg Tost

Laboratory for Epigenetics \& Environment, Centre National de Génotypage, CEA-Institut de Génomique, Evry, France Author for correspondence: tost@eng.fr

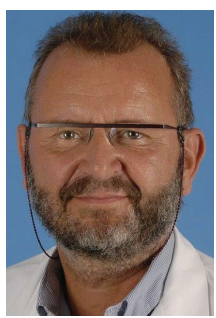

Steffen Gay

Center of Experimental Rheumatology, University Hospital Zurich, Switzerland Author for correspondence:

Steffen.Gay@usz.ch

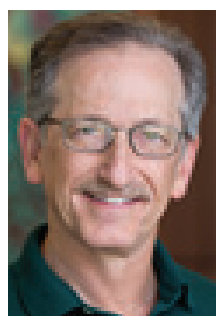

Gary Firestein

Division of Rheumatology, Allergy \& Immunology, University of California San Diego School of Medicine, La Jolla, CA, USA

Author for correspondence: gfirestein@ucsd.edu

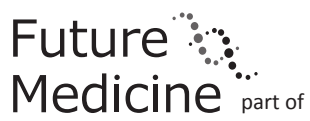


tions. In this issue, Reedquist and colleagues summarize the current state of knowledge on changes in histone acetylation in rheumatoid arthritis [5]. This field is currently of great interest for exploring new therapeutic avenues for the management of rheumatic and inflammatory diseases due to the availability of a number of potentially therapeutic molecules including histone deacetylase and bromodomain and extraterminal (BET) protein inhibitors. BET proteins have been reported to regulate the expression of a large number of genes and play a functional role in controlling inflammation. In this special issue Durham and colleagues show through work in cell lines and animal models that BET proteins can reduce immunoglobulin transcription and production pointing to an important role of BET proteins in (auto)antibody production [6]. The potential therapeutic possibilities using inhibitors of BET proteins are further detailed in the perspective by Tough \& Prinja summarizing evidence that these inhibitors preferentially influence the expression of disease-associated super enhancers in models of various inflammatory diseases [7]. These treatment regimes already in use for cancer might also provide new treatment avenues for immune-related diseases.

Research is not only about the facts and knowledge, but also about the people performing this research, their motivation and vision. In this special issue we have interviewed Amr Sawalha, who works on the epigenetics of systemic lupus erythematosus [8]. He shares his insightful thoughts on current challenges, recent breakthroughs and where he sees the field of research in a decade from now. His research group also contributes a research article on the identification of agerelated methylation changes in naive $\mathrm{CD}^{+}$cells, which might contribute to the predisposition to autoimmunity [9]. Tsokos and coworkers critically review the ample literature on the changes of DNA methylation in lupus as well as their potential as biomarkers or role in personalized therapeutic patient management [10].

\section{"The immune system with its high demands on plasticity and reactivity toward internal and external cues is a paradigm for epigenetic regulation.}

Bergmann and Distler reviewed in detail a third autoimmune disease, systemic sclerosis, in this special issue and describe how epigenetic changes participate in the course of the disease driving fibrosis [11]. Finally, low-level inflammation is present in many complex diseases including atherosclerosis. The research work by de Winther and colleagues shows how the lysine demethylase $6 \mathrm{~B}$ is at least partly responsible for the formation of atherosclerotic lesions and drives the profibrotic gene expression signature in animal models [12].
While autoimmune diseases are characterized by a dysregulated immune response to self-protein, allergies involve an aberrant immune response to exogenous molecules, thus, representing two facets of immune deregulation. The concept that asthma is largely determined at birth and modulated by prenatal and early postnatal environmental exposures is discussed in detail in the perspective by deVries and Vercelli [13]. Tost and coworkers provide a detailed review on the epigenetics of allergic diseases including respiratory and food allergies and summarize which treatment protocols for allergic diseases might act either directly or indirectly through epigenetic mechanisms. They also provide a critical overview on the challenges of investigating the epigenetics of allergies and provide an overview on published EWAS in allergic diseases [14]. This overview is complemented in this issue by a new EWAS performed by Johansson et al. on over 700 allergic individuals investigating genome-wide associations of DNA methylation with total and allergen-specific IgE levels [15].

Inflammatory bowel disease is probably the inflammatory disease entity with the best studied epigenetic changes with a large number of studies already published. Nonetheless, a number of challenges remain, which are fully outlined in the review by Kellermayer and coworkers [16].

Although, the field of epigenetic research in immunerelated diseases is still in its infancy, progress has been fast. However, many challenges lie ahead including the heterogeneous presentation of most diseases, the access to and study of relevant tissues as well as questions on the cause or consequence of the observed changes in inflammation. The recent technological advances allowing to work with less (and better defined) material and to assess the functional importance of epigenetic changes using, for example, epigenome engineering technologies will allow the field to advance and accelerate further.

We hope you enjoy reading the contributions in this special issue of Epigenomics and very much welcome your input and feedback for future features and articles.

\section{Financial \& competing interests disclosure}

The authors have no relevant affiliations or financial involvement with any organization or entity with a financial interest in or financial conflict with the subject matter or materials discussed in the manuscript. This includes employment, consultancies, honoraria, stock ownership or options, expert testimony, grants or patents received or pending, or royalties.

No writing assistance was utilized in the production of this manuscript. 


\section{References}

1 Ecker $S$, Beck $S$. Epigenetic variation taking center stage in immunological research. Epigenomics 9(4), 375-373 (2017).

2 Doody KM, Bottini, Firestein GS. Epigenetic alterations in rheumatoid arthritis fibroblast-like synoviocytes. Epigenomics 9(4), 479-492 (2017).

3 Frank-Bertoncelj M, Klein K, Gay S. Interplay between genetic and epigenetic mechanisms in rheumatoid arthritis. Epigenomics 9(4), 493-504 (2017).

4 Glossop JR, Nixon NB, Emes RD et al. DNA methylation at diagnosis is associated with response to disease-modifying drugs in early rheumatoid arthritis. Epigenomics 9(4), 419-428 (2017).

5 Angiololli C, Baeten D, Radstake TR, Reedquist KA. The acetyl code in rheumatoid arthritis and other rheumatic diseases. Epigenomics 9(4), 447-461 (2017).

6 Shim JM, Lee JS, Russell KE et al. BET proteins are a key component of immunoglobulin gene expression. Epigenomics 9(4), 393-406 (2017).

7 Tough DF, Prinjha RK. Immune disease-associated variants in gene enhancers point to BET epigenetic mechanisms for therapeutic intervention. Epigenomics 9(4), 573-584 (2017).

8 Sawalha AH. Interview with Amr H Sawalha: epigenetics and autoimmunity. Epigenomics 9(4), 379-382 (2017).
9 Dozmorov MG, Coit P, Maksimowicz-McKinnon K, Sawalha AH. Age-associated DNA methylation changes in naive $\mathrm{CD}^{+} \mathrm{T}$ cells suggest an evolving autoimmune epigenotype in aging T cells. Epigenomics 9(4), 429-445 (2017).

10 Hedrich CM, Mäbert K, Rauen T, Tsokos GC. DNA methylation in systemic lupus erythematosus. Epigenomics 9(4), 505-525 (2017).

11 Bergmann C, Distler JHW. Epigenetic factors as drivers of fibrosis in systemic sclerosis. Epigenomics 9 (4), 463-477 (2017).

12 Neele AE, Prange KHM, Hoeksema MA et al. Macrophage $\mathrm{Kdm} 6 \mathrm{~b}$ controls the pro-fibrotic transcriptome signature of foam cells. Epigenomics 9(4), 383-391 (2017).

13 DeVries A, Vercelli D. The neonatal methylome as a gatekeeper in the trajectory to childhood asthma. Epigenomics 9(4), 585-593 (2017).

14 Potaczek DP, Harb H, Michel S, Alashkar, Alhamwe B, Renz $\mathrm{H}$, Tost J. Epigenetics and allergy - from basic mechanisms to clinical applications. Epigenomics 9(4), 539-571 (2017).

15 Ek WE, Ahsan M, Rask-Andersen M et al. Epigenome wide DNA methylation study of $\operatorname{IgE}$ concentration in relation to self-reported allergies. Epigenomics 9(4), 407-418 (2017).

16 Kellermayer R. Epigenetic and other challenges in inflammatory bowel diseases. Epigenomics 9(4), 527-538 (2017). 